\title{
Critical Infrastructure Dependencies 1-0-1
}

\author{
H.A.M. Luiijf, A.H. Nieuwenhuijs, and M.H.A. Klaver
}

\begin{abstract}
Most of our critical infrastructures consist of complex systems-of-systems that provide services or products. The coupling mechanism between the chained systems in such complex systems of systems is dependencies. Dependencies may propagate cascading failures. Most studies on dependencies in infrastructures are based on either an economic model or on a graph model. These regard dependencies mostly as an available or unavailable commodity. In this paper we describe a model view on dependencies showing why dependencies in reality are far more complex than some of the models suggest. At the same time, we will present on-going work on how often serious cascading effects between various critical infrastructures occur based upon an empirical analysis of collected data.
\end{abstract}

\section{INTRODUCTION}

$\mathrm{C}$ RITICAL infrastructure (CI) dependencies are of high interest to society due to the possibility of cascading effects that may seriously harm society as a whole [1]. Despite this high interest, we noticed that most methodologies for analysis of infrastructure dependencies have a limited scope and, even worse, do not recognize the simplifications made in the model or the limitations this has on the applicability of the model.

From our earlier work in CI dependency analysis [2]-[5], matched with events from our $\mathrm{CI}$ incident database covering $\mathrm{CI}$ failures worldwide [6], we derived the understanding of the basic dependency mechanisms. These are described in this paper in a methodological way. We will describe the most important aspects of these mechanisms. We have constructed a database containing publicly available reports on major cascading effects in critical infrastructures all over the world. Based on the data of those events we will illustrate aspects of the model and present an analysis about most common cascading effects.

\section{DEFINITIONS}

A critical infrastructure (CI) consists of those assets and parts thereof which are essential for the maintenance of critical societal functions, including the supply chain, health, safety, security, economy or social well-being of people [7].

Manuscript received at April $21^{\text {th }}, 2008$.. This research was partly funded by the EU Commission as part of the $6^{\text {th }}$ framework programme project IRRIIS under contract number FP6-2005-IST-4 027568 and partly funded by the Netherlands Next Generation Infrastructure (NGI) programme.

Eric Luiijf, Albert Nieuwenhuijs, and Marieke Klaver are with TNO Defence, Security and Safety, The Hague, The Netherlands. phone: +3170 3740312, fax: +31 70 3740642, e-mail: \{eric.luiijf, albert.nieuwenhuijs, marieke.klaver\}@tno.nl
Dependency is the relationship between two products in which one product is required for the generation of the other product. Interdependency is the mutual dependency of products [8]

A product is the tangible or intangible produce as a result of direct human activity or machines or systems.

\section{SHORTFALLS OF EARLIER WORK}

When matching our almost daily increasing database on major CI failure incidents and the cascading events resulting from those incidents, we identified shortfalls in other works on dependencies [9]-[13]:

- Confusion between common cause vulnerabilities (e.g., an earth quake causing simultaneous, but unrelated effects in two critical infrastructures) and dependencies.

- Lack of recognition of the multi-dimensional nature of dependencies. These will be explained below.

\section{SYSTEM APPROACH TO MODEL DEPENDENCIES}

Our approach views a CI as a process which requires one or more input(s) for the process (Fig. 1). Below we consider the quality and response elements of the dependencies (or system inputs) and the system response functions.

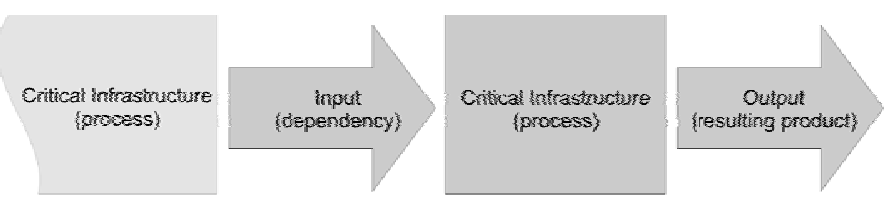

Fig. 1..CI dependencies (process view)

\section{A. Quality}

Most dependency models implicitly presume a total (physical or logical) availability or unavailability of an input. In reality, dependency can be multidimensional as a dependency on a service or commodity is characterized by various quality aspects and levels thereof. E.g., quantity of food or volume of water, information integrity, the temperature of water required for cooling or processing, gas pressure and gas caloric value, voltage, frequency, and biological and chemical purity. When we refer to quality of input(s) or output(s) below, all the abovementioned quality aspects are implicitly included.

\section{B. Response}

A dependency is also characterized by the response function that describes the CI output behavior in response to changes in its input(s). The response function depends both on the 
changes in input(s) - input-response - and on the temporal behavior after a change in the input(s): time-response:

\section{1) Input-response}

We distinguish two types of input-response: (1) the functional behavior describing how the output is related to the dependencies when the dependency quality is or qualities are deteriorating, and (2) the functional behavior describing how the process output is related to dependency qualities when deteriorated input(s) are recovering. Note that this behavior can be asymmetric and even may lead to a lower output level than before the deterioration.

\section{2) Time response}

We can model the time-response functional behavior as a combination of four aspects:

- The time period between the moment the quality of one or more inputs start changing (deteriorating or recovering) and the moment this leads to a (quality) change of the CI output.

- The extent to which the quality of output changes as a function of time in reaction to such a change: the decay and recovery behavior functions. For example: pressure loss in pipelines when the compression process fails and demand continues.

- The differential aspect of dependencies where the quality of output is a consequence of the speed of change of quality of an input. An example of this effect is stockpiling after a steep price increase of a critical commodity like diesel fuel.

- The integrating aspect of dependencies where the speed of change of quality of output is a consequence of the quality of inputs. For example, a crop receiving too little water will yield less and less each day until it finally dies.

\section{State of Operation}

We realized that most of the current dependency models neglect to recognize multiple states of operation (Fig. 2). We discriminate in four distinct states:

- Normal: the state in which a CI operates under normal operational conditions.

- Stressed: state in which a CI operates when special measures have to be taken to keep the operation under control.

- Crisis: this is the state where the operation is out of control.

- Recovery: this is the state where the operation is again brought under control but has not yet been restored to the normal state.

From our understanding of dependencies we see that most cascading failures occur due to operators not realizing that they have different sets of dependencies in each of these states of operation. In each of these states, the set of products and services on which the output depends may be different as well as the extent to which the quality of output depends on the quality of inputs (Fig. 3).

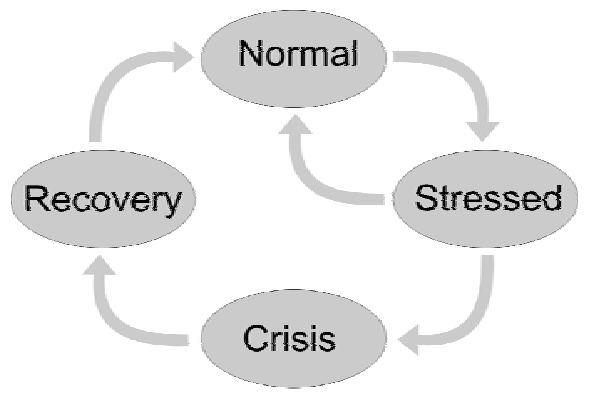

Fig. 2. CI states and the state transition diagram

For instance, fixed telephony may be selected as a fall-back communication option for the automated monitoring of the state of remote parts of the critical infrastructure process. When the automated remote data collection is disrupted, the company switches to stressed mode and the telephony switchboard becomes a very essential part of operation. Such a stressed mode dependency may require special protection measures such as an emergency power supply.

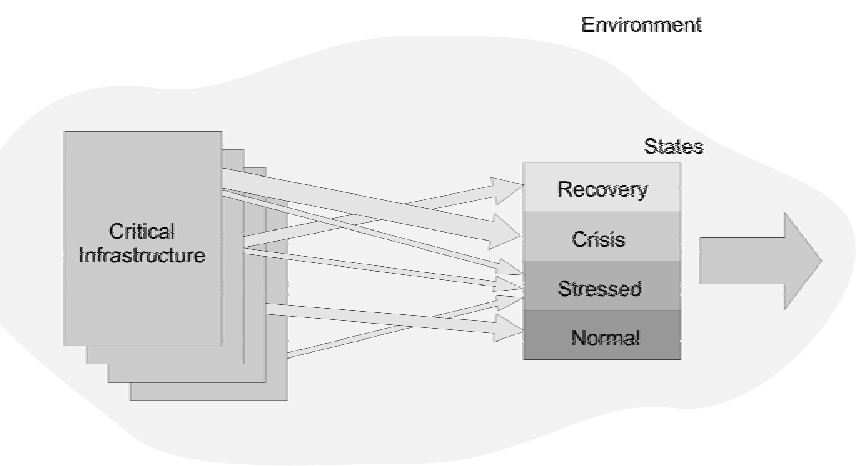

Fig. 3. States and environment as part of the dependency model

\section{Environmental factors}

In trying to understand why certain dependency driven events were considered serious and others not, we realized that a set of environmental factors outside the scope of CI influence the impact of dependencies. These factors can worsen or alleviate dependencies, change their response or even create new dependencies. Some examples:

- In case of a low ambient temperature, the efficiency of a cooling tower increases and decreases the dependency on surface water for cooling.

- A power failure taking out traffic lights is likely to cause hours of chaos when it happens during rush hour. On a Sunday afternoon, it is probably just a nuisance.

- The amount of gas needed for heating is high in winter time and may be next to nil in summer time. 


\section{ANALYSIS OF REAL-WORLD CI DEPENDENCIES}

In order to be able to analyze the way dependencies occur in real life, a database was constructed of CI outages and their cascading effects [6] with over $2590 \mathrm{CI}$ disruption events which have occurred in 107 nations and 'cyberspace'. It contains a collection of publically available outages which concerned around 10.000 or more customers. Initially, on-going analysis of the data shows some surprising results.

First of all, cascading effects due to dependencies appear to be more common than expected. The number of multiple cascading events, however, is lower than some models suggest. The deepest cascades reported are weak cascades of three dependencies deep.

The energy and telecommunication sectors are the main drivers; other sectors are more the 'victim' of dependencies than cascade initiators. Interdependencies hardly seem to occur (two out of 1046 cases) when considering the major effects of CI disruptions as reported by international news sources.

\section{CONCLUSION AND WAY FORWARD}

Increased understanding of $\mathrm{CI}$ dependencies requires new models to model CI dependencies, where an explicit choice is made which aspects of dependencies have to be included in order to meet the anticipated goal of the model. These aspects include various qualities and levels thereof, input and time response elements, multiple states of operation, and environmental factors. The environmental factors comprise a large set of influences on dependencies, some of which are not well understood yet such as season of the year, time of day, temperature, weather, and psychological preconditioning. Further research on the nature of these environmental factors and a possible taxonomy is recommended.

Initial analysis based upon incident data collected from news and other public sources show that cascades occur more often than expected, but cascade less deep than earlier models suggest. Interdependencies hardly occur at a level that is visible enough to be reported by the media and official authorities.

\section{REFERENCES}

[1] European Commission, Critical Infrastructure Protection in the fight against terrorism, $\operatorname{COM(2004)~} 702$ final, Communication from the Commission to the Council and the European Parliament, Brussels, 2004.

[2] H.A.M. Luiijf, H.H. Burger, M.H.A. Klaver, Q. Boone, C. Poppe and M. van Eck, Critical Infrastructure Protection in the Netherlands: Quick-scan on Critical Products and Services, Ministry of the Interior and Kingdom Relations, The Hague, The Netherlands, 2003.

[3] A.H. Nieuwenhuijs, I.M. Paarlberg, J.M.B. Ribbens, H.A.M. Luiijf, Risk Analysis, IRRIIS Deliverable D1.2.2, January 2007.

[4] P. Koponen, H.A.M. Luiijf, H. Pentikäinen, W. Schmitz, T. Uusitale, Report of scenario, threat and vulnerability refinement, IRRIIS Deliverable D1.2.3, March 31, 2007.

[5] A.H. Nieuwenhuijs, R.D. Verstoep, Methods for (inter)dependency analysis, EURAM project deliverable, The Hague, The Netherlands, December 2007.
[6] TNO's database on critical infrastructure incidents and cascading events, version 160, August 22, 2008.

[7] European Commission, Proposal for a Directive of the Council on the identification and designation of European Critical Infrastructure and the assessment to improve their protection, $\operatorname{COM(2006)~} 787$ final, Communication from the Commission to the Council and the European Parliament, Brussels, 12 December 2006.

[8] ACIP consortium, Analysis and Assessment for Critical Infrastructure Protection (ACIP) final report, EU/DG Information Society and Media, Brussels, Belgium, 2003.

[9] S.M. Rinaldi, J. Peerenboom, T. Kelly, Complexities in identifying, Understanding, and Analyzing Critical Infrastructure Dependencies, Special issue IEEE Control Systems Magazine on "Complex Interactive Networks", December 2001.

[10] N.K. Svendsen, S.D. Wolthuysen, Connectivity models of interdependency in mixed-type critical infrastructure networks, Information Security Technical Report Vol 12, No 1, pp 44-55, Elsevier, ISSN 1363-4127.

[11] D. Mendonça, E.E. Lee, W.A. Wallace, Impact of the 2001 World Trade Center attack on Critical Interdependent Infrastructures, IEEE, 2004.

[12] H-S.J. Min, W. Beyeler, Th. Brown, Y.J. Son, A.T. Jones, Toward modeling and simulation of critical national infrastructure interdependencies, HE Transactions (2007)39, 51-71.

[13] D.D. Dudenhoeffer, M.R. Permann, and M. Manic, "CIMS: A Framework For Infrastructure Interdependency Modeling And Analysis.", Proceedings of the IEEE Winter Simulation Conference, 2006. WSC 06. Volume, Issue 3, 6 Dec. 2006, Page(s): 478 - 485, 2006. 